\title{
Nanoscale mapping of optically inaccessible bound-states-in-the-continuum
}

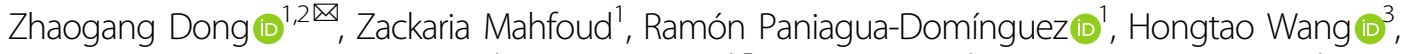 \\ Antonio I. Fernández-Domínguez ${ }^{4}$, Sergey Gorelik',5, Son Tung Ha', Febiana Tjiptoharsono', Arseniy I. Kuznetsov (1)', \\ Michel Bosman (10) ${ }^{1,2 \otimes}$ and Joel K. W. Yang (1) ${ }^{1,3 凶}$
}

\begin{abstract}
Bound-states-in-the-continuum (BIC) is an emerging concept in nanophotonics with potential impact in applications, such as hyperspectral imaging, mirror-less lasing, and nonlinear harmonic generation. As true BIC modes are nonradiative, they cannot be excited by using propagating light to investigate their optical characteristics. In this paper, for the 1st time, we map out the strong near-field localization of the true BIC resonance on arrays of silicon nanoantennas, via electron energy loss spectroscopy with a sub-1-nm electron beam. By systematically breaking the designed antenna symmetry, emissive quasi-BIC resonances become visible. This gives a unique experimental tool to determine the coherent interaction length, which we show to require at least six neighboring antenna elements. More importantly, we demonstrate that quasi-BIC resonances are able to enhance localized light emission via the Purcell effect by at least 60 times, as compared to unpatterned silicon. This work is expected to enable practical applications of designed, ultra-compact BIC antennas such as for the controlled, localized excitation of quantum emitters.
\end{abstract}

\section{Introduction}

Bound-states-in-the-continuum (BIC) is a fascinating concept that has its origins in quantum mechanics in $1929^{1}$. Counterintuitively, optical modes with energies higher than the potential wells, i.e., in the freely propagating continuum, can still be localized and bound in space when these wells are appropriately designed ${ }^{1}$. This BIC concept was first demonstrated experimentally in semiconductor heterostructures in $1992^{2}$, based on earlier theoretical predictions ${ }^{3,4}$. To date, the concept of BIC has been generalized as a wave phenomenon into various fields, such as acoustics ${ }^{5,6}$, microwave physics ${ }^{7,8}$, and optics $^{9-13}$. BIC in optics gives remarkably narrow

\footnotetext{
Correspondence: Zhaogang Dong (dongz@imre.a-star.edu.sg) or Michel Bosman (msemb@nus.edu.sg) or Joel K.W. Yang (joel_yang@sutd.edu.sg) 'Institute of Materials Research and Engineering, A*STAR (Agency for Science, Technology and Research), 2 Fusionopolis Way, \#08-03 Innovis, 138634 Singapore, Singapore

${ }^{2}$ Department of Materials Science and Engineering, National University of

Singapore, 9 Engineering Drive 1, 117575 Singapore, Singapore

Full list of author information is available at the end of the article

These authors contributed equally: Zhaogang Dong, Zackaria Mahfoud
}

resonances that are controlled by the design of nanostructures, such as dielectric gratings ${ }^{9}$, arrays of coupled waveguides $^{10,11}$, layered nanoparticles ${ }^{12}$, and photonic crystal slabs $^{13}$. The general principle of BIC is the complete suppression of radiative losses in the far-field by total destructive interference of radiation from the charge-current configuration associated with the mode $^{14,15}$. These BIC modes have energies above the light line, hence the "continuum" denomination, but are completely "dark" in the sense that they do not couple to radiative modes in free-space but are instead bound to the structures that support them.

Recently, BIC has been explored in nanophotonics mostly with nanoantenna arrays ${ }^{16-22}$, but in individual resonators as well ${ }^{12,23-25}$. Nanoantenna designs with broken symmetry provide small but sufficient coupling to form quasi-BIC modes that enable far-field optical excitation, while true BIC modes do not couple to the far-field ${ }^{17-22,26}$. As the (quasi-) BIC modes are supported by nanoantennas, controlling their geometry can achieve unidirectional scattering ${ }^{27}$, sensitive hyperspectral

\section{(c) The Author(s) 2022}

(c) (i) Open Access This article is licensed under a Creative Commons Attribution 4.0 International License, which permits use, sharing, adaptation, distribution and reproduction cc) in any medium or format, as long as you give appropriate credit to the original author(s) and the source, provide a link to the Creative Commons license, and indicate if changes were made. The images or other third party material in this article are included in the article's Creative Commons license, unless indicated otherwise in a credit line to the material. If material is not included in the article's Creative Commons license and your intended use is not permitted by statutory regulation or exceeds the permitted use, you will need to obtain permission directly from the copyright holder. To view a copy of this license, visit http://creativecommons.org/licenses/by/4.0/. 
imaging $^{21}$, lasing ${ }^{28-30}$, nonlinear nanophotonics ${ }^{20,24,31,32}$, chirality $^{33}$, bio-sensing ${ }^{21,22,34}$, and topological photonics $^{26,27}$. However, due to the "dark" nature of the true $\mathrm{BIC}$ resonances, optical excitation in the far-field is unable to reveal its mode characteristics. In addition, although it has been demonstrated that a finite antenna array with only $8 \times 8$ elements is able to achieve BIC lasing under far-field optical excitation ${ }^{35}$, no direct characterization technique has determined the characteristic length that is required to setup a BIC mode, or rather how quickly the BIC mode decays as it approaches the edge of a large array.

BIC modes have no coupling to free-space radiation and are therefore optically inaccessible, except for the quasiBIC modes. Experiments that attempt to measure BIC modes will therefore need to be sensitive to both the nearfield and the far-field simultaneously; in this way, the "true", non-radiative BIC modes can be distinguished from the quasi, radiative BIC modes. To this aim, our experiments combine cathodoluminescence (CL) and monochromated electron energy loss spectroscopy (EELS) in a scanning transmission electron microscope (STEM). The nanometer-sized, focused electron beam is used to excite the optical modes in the near-field. The energy transferred in these radiative and non-radiative excitations is measured with EELS, while only radiative losses are measured with $\mathrm{CL}$ in the far-field. A comparison of both EELS and CL spectra distinguishes the lossy and the trapped optical modes, providing an experimental setup to unambiguously characterize true photonic BICs with nanometer spatial precision.

In this work, we provide experimental and theoretical evidence of true BIC resonances that are locally excited by a nanometer electron beam in the STEM and directly probed using EELS and CL spectroscopy. Localized plasmonic optical transitions have been investigated before in the STEM $^{36-39}$. The electron microscope was shown to provide a unique combination of broadband, near-field excitation, wide-range spectroscopy, and ultrahigh spatial resolution, enabling a comprehensive analysis of optical resonances. Here, we use it to probe the BIC mode on designed arrays of silicon nanoantennas. By combining the spectroscopic techniques CL and EELS in the STEM, we provide direct experimental evidence of "true" BIC modes. We place "true" in quotation marks, as our fabricated arrays are necessarily of finite size, while true BIC modes in the strict term are only fully developed in infinitely large arrays. Numerical simulations as well as multipolar decomposition analysis support our claim that quasi- and "true" BIC modes can be distinguished.

\section{Results}

Figure 1a shows the experimental setup for probing the BIC mode, where a high-energy electron beam with a diameter of $\sim 1 \mathrm{~nm}$ in the STEM is focused onto an array of Si nanoantennas, lithographically defined on a 30-nm-thick suspended amorphous $\mathrm{Si}_{3} \mathrm{~N}_{4}$ membrane (see Fig. $\mathrm{S} 1$ and Methods section for fabrication details). When this focused electron beam is positioned near a nanostructure, a fraction of the energy of the electron beam will be used to polarize the free and bound electrons within the material that start to oscillate according to the modes supported by the nanostructures. These resonant modes can subsequently lose their energy to free-space emission, which is measured with $\mathrm{CL}^{40-43}$, as shown previously for metallic nanostructures ${ }^{43-49}$ and silicon nanoantennas ${ }^{50-54}$. All the losses - radiative and non-radiative-that the electron beam encounters in the excitation of the resonant modes are measured experimentally with EELS, a true near-field technique. By combining both CL and EELS spectroscopy

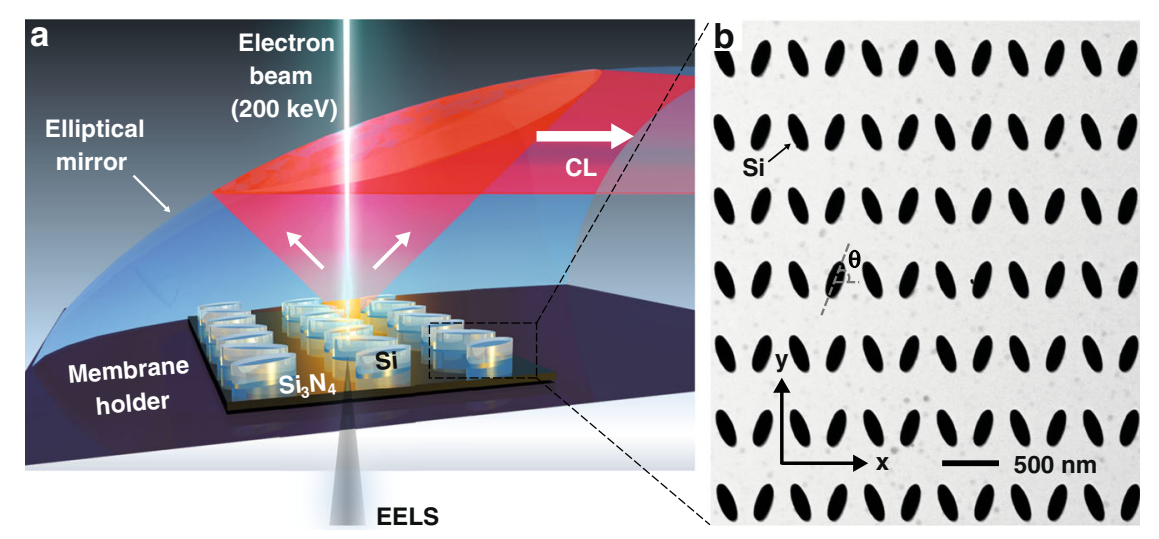

Fig. 1 Experimental setup for exciting and probing bound-states-in-the-continuum (BIC) modes via a sub-1-nm electron beam. a Schematic of the experimental setup in a STEM where a high-energy electron beam focused to $\sim 1 \mathrm{~nm}$ is used to probe an array of Si nanoantennas on a 30-nm thick suspended $\mathrm{Si}_{3} \mathrm{~N}_{4}$ membrane. Complementary measurements of the energy lost by electrons, and energy of emitted photons result in EELS and $\mathrm{CL}$ spectra respectively. $\mathbf{b}$ Bright-field STEM image of an amorphous Si nanoantenna array supporting quasi-BIC modes on a 30-nm-thick $\mathrm{Si}_{3} \mathrm{~N}_{4}$ membrane. "True" BIC occurs when $\theta=90^{\circ}$ 
a

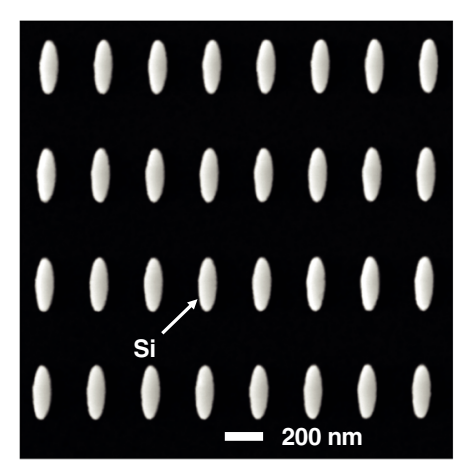

C

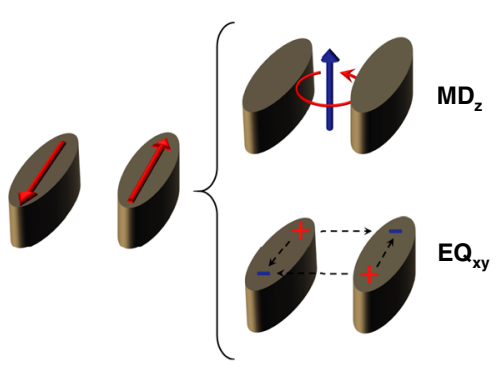

e

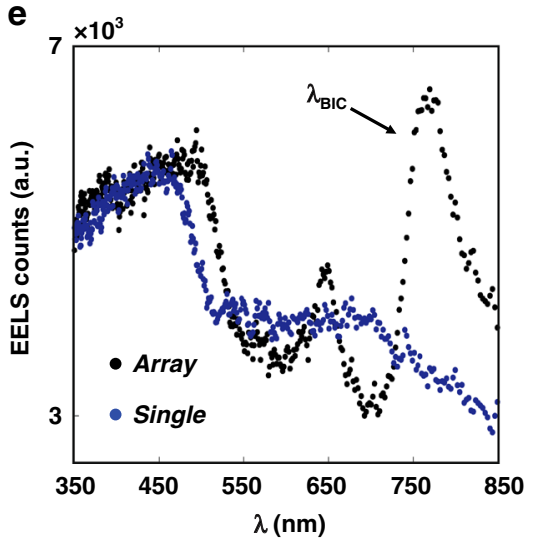

b

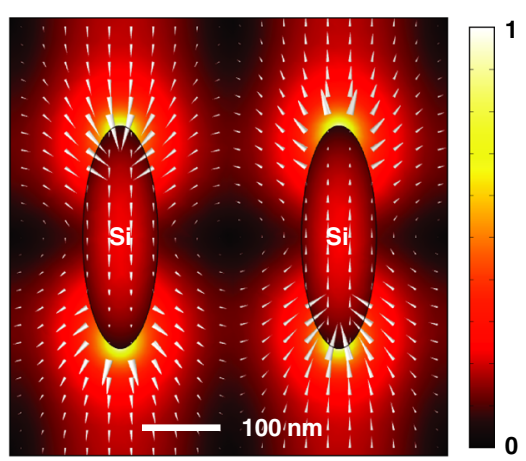

d

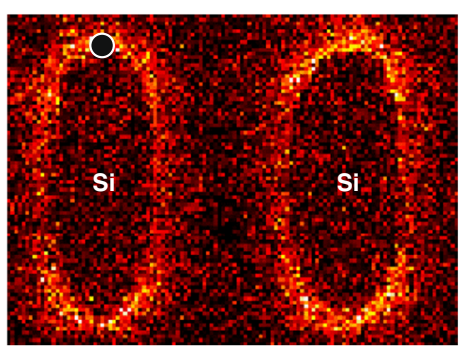

f

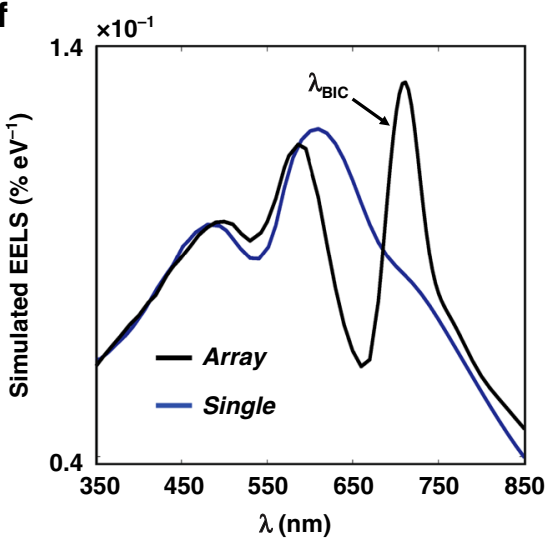

Fig. 2 Probing of a "true" BIC resonance mode by Electron Energy Loss Spectroscopy. a STEM annular dark-field image of a silicon nanoantenna array with elliptic cylinders aligned at a tilt angle $\theta=90^{\circ}$. b Simulated near-field mode pattern of the "true" BIC mode, with arrows indicating the electric field direction; their length being proportional to the amplitude. c Schematic of the multipole moments comprising the "true" $\mathrm{BIC}$ mode: the z-polarized magnetic dipole $\left(\mathrm{MD}_{z}\right)$ and the electric quadrupole $\left(E Q_{x y}\right)$. $\mathbf{d}$ Experimental EELS map of the elliptic cylinder nanoantenna array with the "true" BIC mode resembling the E-field maps in (b). e EELS spectrum (black color) as measured at the position near the tip of the ellipse as indicated in (d) demonstrating the excitation of the "true" BIC mode. For benchmarking, the EELS spectrum measured from an isolated single antenna element of the same size (blue color) clearly shows the missing peak associated with the BIC mode. More information about the single antenna measurements can be found in the Fig. S3. f FEM-simulated EELS spectra for the nanoantenna array (black), showing a BIC resonance, and for a single antenna element (blue), showing no BIC resonance

techniques in the STEM, we will demonstrate the possibility of measuring BIC modes at the nanometer length scale. Our universal approach to distinguish all the bright and dark optical modes has the additional advantage of performing simultaneous STEM imaging with nanometer resolution, to visualize the local sample morphology. A more detailed description of the experimental setup for measuring "true" and quasi-BIC modes is given in the Methods section.
Figure $1 \mathrm{~b}$ shows a STEM image of an array of Si elliptic cylinder nanoantennas that support (quasi-) BIC modes. The designed dimensions for the long-axis and short-axis of the antennas are $286 \mathrm{~nm}$ and $96 \mathrm{~nm}$, respectively, with a pitch size of $562 \mathrm{~nm}$. The tilt angle $\theta$ of the nanoantennas is commonly used to control the degree of symmetric breaking of the coupled nanoantennas ${ }^{19}$, providing farfield optical access to the quasi-BIC mode. As $\theta$ approaches $90^{\circ}$ (see the STEM image in Fig. 2a), the silicon 
nanoantenna array supports a "true" BIC mode that is not optically accessible from the far-field. The near-field pattern of this "true" BIC mode can be found by computing the eigenmodes of the system. Figure $2 b$ shows the simulation results, corresponding to a BIC mode at the Gamma symmetry point at a wavelength of $\sim 720 \mathrm{~nm}$ and a quality factor ( $Q$-factor) of $\sim 90$. Note that the finite bandwidth of the mode is due to the dissipative loses (i.e., the extinction coefficient $k=0.01$ ) of silicon at that wavelength; the measured $n$ and $k$ values for the amorphous silicon film are shown in Fig. S2. This finite $Q$ factor value of the "true" BIC mode is consistent with the ones reported in the literature ${ }^{55}$, in which they analyzed the limited Q-factor of BICs imposed by absorption in silicon. In addition, the near-field oscillation characteristic of this BIC mode is shown in the supporting video file "NF_BIC.gif", where the neighboring unit cells are oscillating in phase when $k=0$. The schematic in Fig. 2c shows the equivalent multipole moments that give rise to the BIC, consisting of a $z$-polarized magnetic dipole $\left(\mathrm{MD}_{z}\right)$ and an electrical quadrupole $\left(\mathrm{EQ}_{x y}\right)$. Due to its non-radiative nature, this "true" BIC resonance mode can neither be excited nor measured using far-field techniques $^{23}$. In our experimental setup, the electron energy losses measured with monochromated EELS would detect this BIC mode in the near-field. On the other hand, in situ CL measurements that collect emitted light will allow us to determine if this mode can be optically detected in the far-field.

EELS measurements of a "true" BIC mode are presented in Fig. 2. The measured EELS BIC map in Fig. 2d and EELS spectra in Fig. 2e show clear evidence of a BIC mode in the silicon antenna array, while this mode is strikingly absent when EELS is measured on individual antennas that are not part of an array, confirming the interpretation of the array effect. To corroborate these experimental observations, Fig. 2f presents finite-element-method (FEM)-based COMSOL simulations of EELS spectra for a single antenna and for antennas in an array (see Methods). The simulation verifies the observed difference between the antenna array and the single element. Moreover, the first row in Fig. 3b presents optical reflectance measurements under $x$-polarized incidence condition (see the $x-y$ coordinate in the STEM image in Fig. 1b), where no BIC resonance feature is observed at $\sim 720 \mathrm{~nm}$ in the case of a $90^{\circ}$ tilt angle due to its nonradiative nature. Final evidence is provided in Fig. 3c where no BIC resonance is observed in the $\mathrm{CL}$ far-field emission, though the EELS data in Fig. 3a clearly show that the mode is excited by the electron beam. This is direct verification of the non-radiative nature of this "true" BIC mode.

In an attempt to distinguish the "true" bound states from the emissive quasi-BIC resonances, we patterned symmetry-broken arrays of the same nanoantennas by changing the tilt angle $\theta$ of the nanoantennas from $90^{\circ}$, to $80^{\circ}$, and $70^{\circ}$. Figure $3 \mathrm{a}$ presents the measured EELS spectra showing both the "true" $\mathrm{BIC}$ resonance at $\theta=90^{\circ}$ and the quasi-BIC resonances at $\theta=80^{\circ}$ and $\theta=70^{\circ}$. The quasi-BIC mode now becomes visible both in the reflectance spectra and in the CL emission spectra, as shown in Fig. 3b, c, while this mode is not present for the "true" BIC at $\theta=90^{\circ}$. For completeness, the simulated reflectance spectra, the EELS maps for the quasi-BIC mode at $\theta=$ $70^{\circ}$, and the substrate effects due to varying $\mathrm{Si}_{3} \mathrm{~N}_{4}$ membrane thicknesses are shown in Figs. S4-6, respectively. In addition, Fig. S7 presents angle-resolved reflectance spectra under $x$-polarization and it shows that the true BIC modes for the elliptic cylinder nanoantenna pairs with a tilt angle $\theta$ of $90^{\circ}$ are inaccessible by far-field propagating light.

For the case when $\theta=70^{\circ}$, the quasi-BIC mode is particularly strong in CL emission, and has a full-widthat-half-maximum (FWHM) of $\sim 28 \mathrm{~nm}$ (see Fig. S8 for details). The local field distribution for this quasi-BIC resonance is given in Fig. 3d, showing a $\sim 23$-fold local electric field enhancement factor. Figure $3 e$ presents the corresponding multipolar decomposition, showing how electric dipole (ED), magnetic dipole (MD), electric quadrupole (EQ) and magnetic quadrupole (MQ) contributions are excited. The magnetic dipole is directed along the $z$-direction $\left(\mathrm{MD}_{z}\right)$, while the electric dipole lies in-plane along the $x$-direction $\left(\mathrm{ED}_{x}\right)$, allowing the in- and out-coupling of the incident plane-wave radiation. Therefore, the nano-optical setup of STEM-EELS in combination with $\mathrm{CL}$ is able to differentiate and characterize both non-radiative "true" BIC resonances as well as the radiative quasi-BIC resonances.

When the antenna tilt angle $\theta$ is changed from $80^{\circ}$ to $70^{\circ}$, the $Q$ factor will be reduced due to the increased symmetry breaking ${ }^{19}$. This phenomenon is confirmed on the experimentally measured $\mathrm{CL}$ emission as shown in Fig. 3c, with a detailed analysis in Figs. S8 and S9. It shows that when the tilt angle $\theta$ is changed from $80^{\circ}$ to $70^{\circ}$, the $Q$ factor is reduced from $\sim 32$ to $\sim 26$ with the corresponding FWHM being changed from $\sim 23 \mathrm{~nm}$ to $\sim 28 \mathrm{~nm}$.

There exists a slight resonance shift between the measured EELS/CL spectra in Fig. 3a, c, with respect to the measured optical reflectance in Fig. 3b. This slight resonance shift originates from imperfections introduced in the nanofabrication process and from the difference in measurement principles. Firstly, the BIC antenna array was fabricated on 30-nm-thick, suspended $\mathrm{Si}_{3} \mathrm{~N}_{4}$ membranes that are not perfectly flat due to its ultra-thin nature. During fabrication, this curvature will introduce slight local variations in shapes and dimensions of the fabricated silicon BIC nanoantenna arrays. Secondly, the reflectance spectrum in Fig. $3 \mathrm{~b}$ is measured from a 

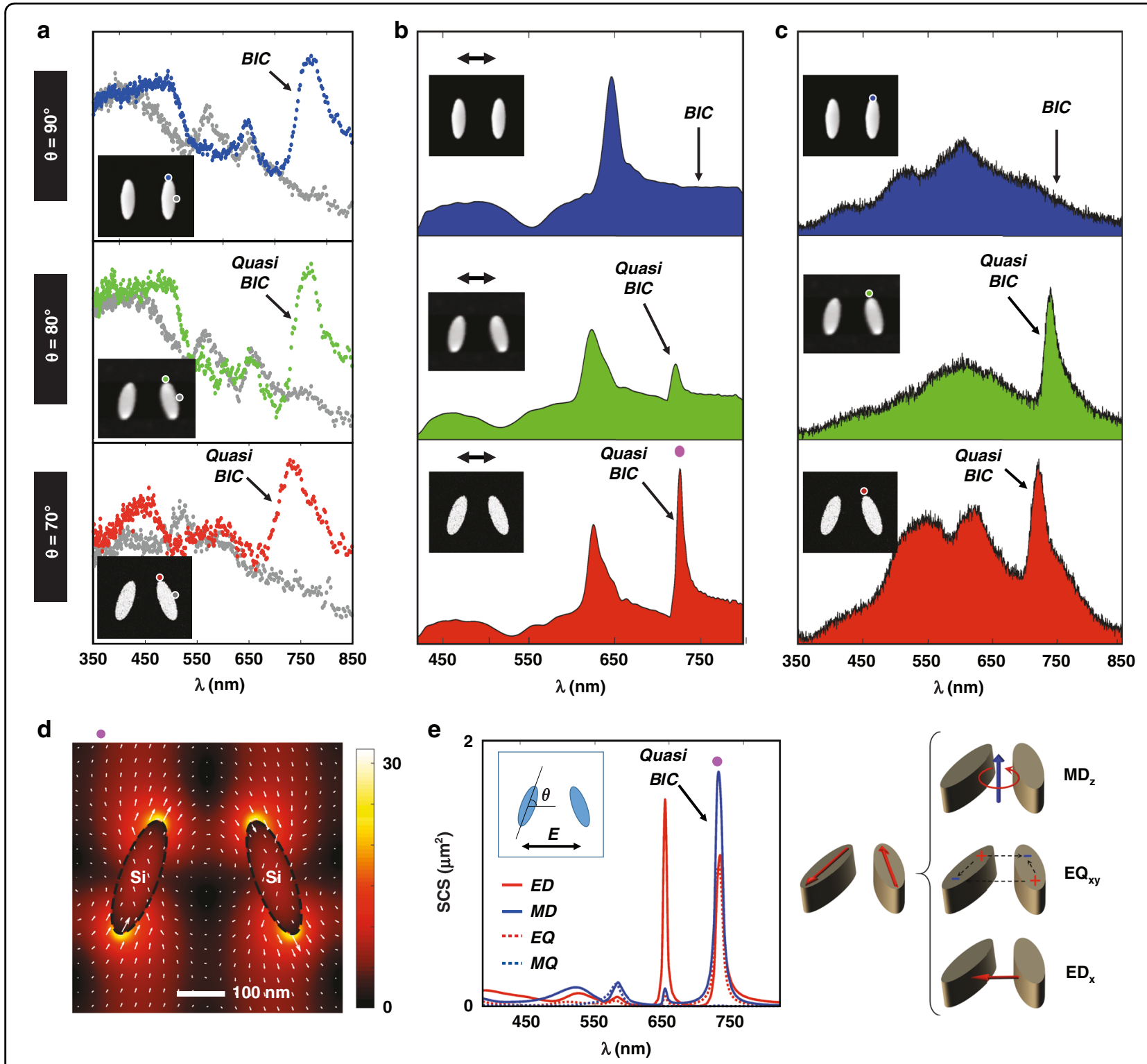

Fig. 3 Evolution of CL and EELS spectra for nanoantenna arrays with different tilt angles. a Measured EELS spectra, $\mathbf{b}$ reflectance spectra under $x$-polarized incidence condition, and $\mathbf{c} \mathrm{CL}$ spectra from the nanoantenna array with different tilt angle $\theta$ of $90^{\circ}, 80^{\circ}$, and $70^{\circ}$. $\mathbf{d}$ Spatial distribution of the electric field $|\boldsymbol{E}|$ at the quasi-BIC resonance at $\sim 720 \mathrm{~nm}$ for the nanoantenna array with a tilt angle $\theta$ of $70^{\circ}$. The arrows denote the electric field direction, the length of the arrows being proportional to the amplitude. e Multipolar decomposition of the scattering cross-section (SCS) and corresponding schematics of the multipoles excited at the quasi-BIC mode

large array of $130 \times 130$ elements, making this far-field measurement less sensitive to local variations in antenna size and shape.

One important feature of the quasi-BIC resonance in nanoantenna arrays is that it is a collective array mode, the formation of which involves neighboring nanoantenna elements. To show this collective effect, we investigate the coherent interaction length of the quasi-BIC resonance based on the $\mathrm{CL}$ characterization results in a $\mathrm{Si}$ nanoantenna array. No EELS mapping was used here, as the monochromated EELS signal is much weaker and thus requires extremely long data acquisition times for such a large number of antennas. The $\mathrm{CL}$ mapping was carried out on a Si nanoantenna array containing $130 \times$ 130 antenna elements, the corner of which is shown in the STEM high-angle annular dark-field (HAADF) image in Fig. 4a, where the Si nanoantenna array has a tilt angle $\theta$ of $70^{\circ}$.

Figure $4 \mathrm{~b}$ was obtained by scanning the electron beam in the white dotted rectangle of Fig. $4 \mathrm{a}$ and recording a CL spectrum at each pixel in the scan. This threedimensional $\mathrm{CL}$ data set is then stored and processed as 


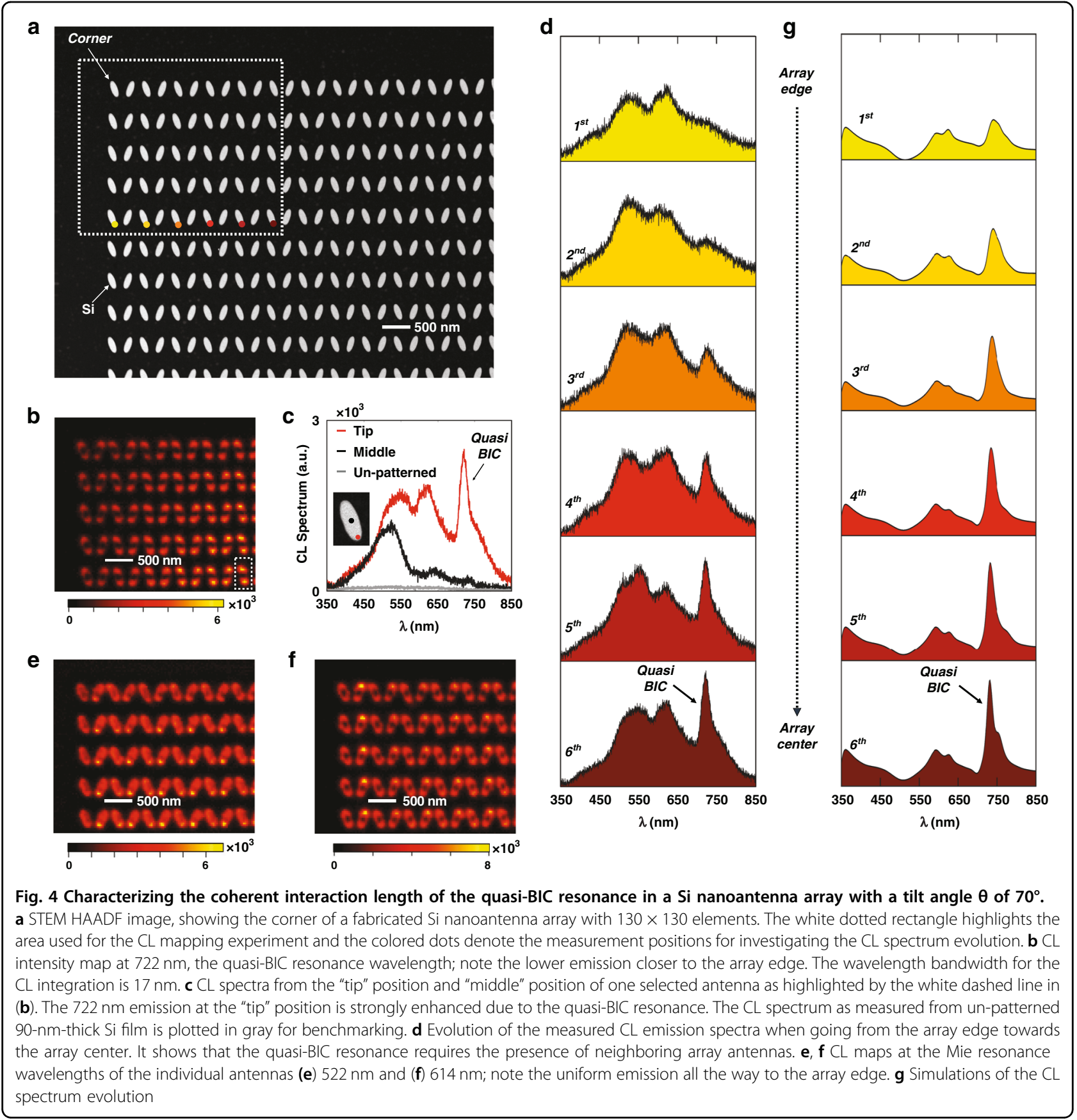

described in the Methods section, resulting in Fig. 4b the spatial distribution of the CL emission at the quasi-BIC resonant wavelength of $\sim 722 \mathrm{~nm}$, using an integration bandwidth of $17 \mathrm{~nm}$. Figure 4c presents the CL spectra measured from a single antenna element indicated in the white dashed rectangle in Fig. $4 \mathrm{~b}$. It shows that the CL emission measured at the tip position of the silicon nanoantenna (in red) is significantly different from the CL spectrum measured at the middle position (in black); these respective measurement positions are indicated on the inset STEM image in Fig. 4c. For comparison, the graph also plots the CL emission measured from a $90-\mathrm{nm}$ thick, unpatterned Si film; its much lower CL intensity is measured to give a 63 times weaker emissivity as compared to the Si array.

The CL map in Fig. 4b gives direct visual evidence of the coherent interaction length of the quasi-BIC resonance due to the collective antenna array effect. As seen there, the CL emission at the quasi-BIC ( $722 \mathrm{~nm}$ wavelength) is very weak at the edge of the antenna array, and it becomes 
stronger when the electron beam moves towards its center. This edge effect becomes even more obvious when we plot the CL spectra as a function of the distance to the edge of the array, shown in Fig. 4d. The 1st antenna element at the array edge shows no sign of the quasi-BIC resonance at all. Our CL experiments demonstrate that the quasi-BIC mode is only fully established after at least six antenna elements into the array; the outer six elements only exhibit a partially established quasi-BIC mode. This observation is in stark contrast with the CL map at the Mie resonances of the nanoantennas shown in Fig. 4e, f. As these resonances are supported by individual antennas, we observe no emission reduction or enhancement as a function of location in the nanoantenna array.

To corroborate this understanding, Fig. S10 presents simulations of the CL emission as enhanced by the quasiBIC resonance. We adopted the approach from Das et al. ${ }^{56}$, performing FDTD simulations with the incident electron beam modeled as an array of $z$-polarized dipole sources with an appropriate phase delay. Here, to account for the spectral broadening effect due to the limited CL spectral resolution, Fig. $4 \mathrm{~g}$ was obtained after the convolution with a normalized Gaussian function (see details in "Methods" section). These simulation results in Fig. S10 and Fig. 4g verify the experimental observations in Fig. 4a-d, showing that the first six antenna elements at the edge of the array only have partially established quasiBIC resonances. In addition, the EELS calculations for nanoparticle arrays of different sizes shown in Fig. S11 indicate that the interaction coherent length for the "true" $\mathrm{BIC}$ resonance is similar to the one extracted from $\mathrm{CL}$ measurements and calculations. This indicates that by tilting the antenna orientation, the modes acquire a radiative character, but their nature remains very similar to that of their fully dark counterpart with $\theta=90^{\circ}$.

\section{Conclusions}

By combining nanoscale electron beam spectroscopy in the STEM, theoretical simulations and multipolar decomposition, we observe "true" photonic BIC on nanostructured Si samples. This localized probing approach provides several distinguishing advantages over conventional optical excitation approaches in the far-field. For instance, the electron beam-induced CL emission is able to spatially map the coherent interaction length of the quasi-BIC resonance, which, in our case, shows that this mode is only partially established in the outer six antenna elements, and only fully formed beyond the sixth element towards the center of the array. When STEM-CL spectroscopy is combined with monochromated STEM-EELS measurements, the "true" $\mathrm{BIC}$ resonance is revealed, even in situations when their excitation is impossible using far-field optical irradiation. Our results provide a general methodology to quantitatively probe the mode formation mechanism for BIC with nanometer spatial precision. It will be broadly applicable to quantify other emerging optical resonances such as localized excitation of quantum emitters, and will provide direct and unique insight into their nature.

\section{Materials and methods \\ Nanofabrication of Si nanostructured on membrane}

Amorphous Si with a thickness of $90 \mathrm{~nm}$ was grown onto a 30-nm-thick $\mathrm{Si}_{3} \mathrm{~N}_{4}$ membrane (Agar Scientific S1711), by using plasma-enhanced chemical vapor deposition (PECVD). Before the electron beam exposure, HSQ resist with a concentration of $2 \%$ wt., diluted in methyl isobutyl ketone (Dow Corning XR-1541-002), was spin coated onto the cleaned substrate at $5 \mathrm{k}$ round-per-minute (rpm), giving a thickness of $\sim 30 \mathrm{~nm}$. The electron beam exposure was carried out with the following conditions: electron acceleration voltage of $100 \mathrm{keV}$, beam current of $200 \mathrm{pA}$, and an exposure dose of $\sim 12 \mathrm{mC} / \mathrm{cm}^{2}$. The sample was then developed by a $\mathrm{NaOH} / \mathrm{NaCl}$ salt solution $(1 \%$ wt./4\% wt. in de-ionized water) for $60 \mathrm{~s}$ and immersed in de-ionized water for 60 seconds to stop the development. Next, the sample was immediately rinsed by acetone, isopropanol alcohol (IPA) and dried by a continuous flow of nitrogen. Si etching was then carried out by using inductively coupledplasma (ICP, Oxford Instruments Plasmalab System 100), with $\mathrm{Cl}_{2}$ gas chemistry at $6{ }^{\circ} \mathrm{C}^{57}$.

\section{CL and EELS in a STEM}

The STEM-CL spectroscopy and STEM-HAADF imaging experiments were carried out with a FEI Titan with the sample at room temperature. The system is equipped with a Schottky emitter and a Wien-type monochromator that was used to disperse and filter the electron beam to a resolution of $65 \mathrm{meV}$. EELS spectra were acquired with a Tridiem HR detector in spectrum imaging mode, using binned gain averaging $^{58}$. The background signal was subtracted by fitting a high-quality 'zero-loss peak' to the measured spectra between 0.5 and $1.0 \mathrm{eV}$, well before the first onset of the experimental peaks. An example of the EELS background fitting is shown in Fig. S12. A Gatan Vulcan (sample holder and light detection system) was used for CL measurements. The electron beam was accelerated at $200 \mathrm{keV}$ with the monochromator being turned off, while the electron beam was focused into a probe approximately $1 \mathrm{~nm}$ in diameter. The CL maps were acquired with a diffraction grating blazed at $500 \mathrm{~nm}$ and a dwell time set to $0.42 \mathrm{~s}$ per spectrum, where the CL spectral resolution is $17 \mathrm{~nm}$, limited by the slit aperture of the spectrometer. Data processing consisted of subtracting the CCD dark-noise read-out and removing strong single-channel 'X-ray' spikes.

\section{Reflectance simulations and multipolar decomposition}

Finite-difference time-domain (FDTD) simulations were carried out using a commercial software (Lumerical 
FDTD Solutions). In order to consider the random deformation of the ultra-thin $\mathrm{Si}_{3} \mathrm{~N}_{4}$ membrane (i.e. $30 \mathrm{~nm}$ in thickness) during the ICP CVD process for growing $\mathrm{Si}$ film, $13 \times 13$ arrays of Si nanoantennas were used with the incident optical field being linearly polarized along $x$-direction at normal incidence condition. A non-uniform meshing technique was applied with a minimum mesh size down to $0.5 \mathrm{~nm}$. The dielectric function of amorphous $\mathrm{Si}$ was taken from the measured $n$ and $k$ values by ellipsometry ${ }^{59}$. Moreover, multipolar decomposition analysis was carried out to analyze the optical modes being excited within the structures, where this multipolar decomposition was implemented by Finite Element Method (FEM) in COMSOL. The detailed formulas of multipolar analysis can be found in the supporting information of the reference ${ }^{59}$.

\section{CL simulations}

The simulation of $\mathrm{CL}$ emission was based on a finitedifference time-domain (FDTD) approach as reported in the literature ${ }^{56}$, where an array of dipole sources is able to simulate the CL emission process. Fig. S10a presents a schematic, using an array of $41 z$-polarized dipole sources distributed with a uniform separation of $50 \mathrm{~nm}$ along the $z$-direction and having a relative phase-shift following the expression $\mathrm{e}^{\mathrm{i} \omega z / \nu}$. Here, $\nu$ denotes the electron speed, $\omega$ denotes the oscillation frequency and $z$ denotes the dipole position $^{56}$. These $41 z$-polarized dipole sources are placed symmetrically with respect to the 30-nm-thickness membrane. In our TEM setup, the acceleration electron voltage is $200 \mathrm{kV}$, and the electrons are traveling at a speed of $\sim 0.695 \mathrm{c}$. To record the CL emission, a monitor is placed at a $z$-plane, which is $500 \mathrm{~nm}$ above the top surface of the $13 \times 13$ elliptic cylinder nanoarray. In order to remove the direct emission of these dipole sources, we take the vectorial subtraction between the total electrical field components with and without the elliptic cylinder nanoarray. After that, a near-to-far transformation was carried out to simulate the collected CL emission within a solid angle of $\left(-15^{\circ}, 15^{\circ}\right)$. Detailed CL simulation results are shown in Fig. S10. In addition, to account for the spectral broadening effect due to the limited spectral resolution for CL measurements, these simulated CL spectra were convoluted with a normalized Gaussian function of $16 \mathrm{meV}$ width to get the simulated CL emission profiles as shown in Fig. 4g.

\section{EELS simulation}

The numerical calculations were performed under the finite-element solver of Maxwell's Equations in frequency domain implemented in COMSOL Multiphysics. The frequency-dependent electron beam propagating along $z$-direction at position $\left(x_{e}, y_{e}\right)$ was simulated by a line current of the form $j(r, \omega)=e \exp \left\{-\frac{i \omega z}{v}\right\} \delta\left(x-x_{e}\right) \delta\left(y-y_{e}\right) \hat{z}$, where $e$ is the electron charge and the electron velocity, $v$, was set in accordance with the experiments. The energy loss probability is then calculated as $A=\Gamma=$ $\frac{1}{\pi \hbar \omega} \int \mathrm{d} z \operatorname{Re}\left\{j^{*}(r, w) E_{\mathrm{SC}}(r, \omega)\right\}$, where $\boldsymbol{E}_{\mathrm{sc}}(\boldsymbol{r}, \omega)$ are the electric fields scattered by the dielectric nanoparticle array $^{40}$. The EELS spectra were calculated by convolving $\Gamma(\omega)$ with a normalized Gaussian function of $65 \mathrm{meV}$ width, mimicking the experimental spectral resolution. Detailed EELS simulations for different array sizes are shown in Fig. S11 for the case $\theta=90^{\circ}$.

\section{Acknowledgements}

The authors would like to acknowledge the funding support from Agency for Science, Technology and Research (A*STAR) SERC Pharos project (grant number 1527300025). In addition, Z.D. and J.K.W.Y. would like to acknowledge the A*STAR AME IRG funding support with the project number A20E5c0093. Z.D. acknowledges support from A*STAR career development award (CDA) with the grant number 202D8088. M.B. acknowledges support from the Singapore Ministry of Education Academic Research Fund Tier 2 (project number MOE2019-T2-1-179). A.I.F.D. was supported by a 2019 Leonardo Grant for Researchers and Cultural Creators, BBVA Foundation. Z.M. acknowledges support from the European Union's Horizon 2020 research and innovation program under grant agreement No 823717 - ESTEEM3. J.K.W.Y. would like to acknowledge the funding from National Research Foundation (NRF) Singapore with the grant numbers NRF-CRP20-2017-0001 and NRF-NRFI06-2020-0005.

\section{Author details \\ ${ }^{1}$ Institute of Materials Research and Engineering, A*STAR (Agency for Science, Technology and Research), 2 Fusionopolis Way, \#08-03 Innovis, 138634 Singapore, Singapore. ${ }^{2}$ Department of Materials Science and Engineering, National University of Singapore, 9 Engineering Drive 1, 117575 Singapore, Singapore. ${ }^{3}$ Singapore University of Technology and Design, 8 Somapah Road 487372 Singapore, Singapore. ${ }^{4}$ Departamento de Física Teórica de la Materia Condensada and Condensed Matter Physics Center, Universidad Autónoma de Madrid, 28049 Madrid, Spain. ${ }^{5}$ Singapore Institute of Food and Biotechnology Innovation, A*STAR (Agency for Science, Technology and Research), 31 Biopolis Way, \#01-02 Nanos, 138669 Singapore, Singapore}

\section{Author contributions}

Z.D. and J.K.W.Y. conceived the concept, designed the experiments and wrote the manuscript with M.B. Z.M. and M.B. performed the cathodoluminescence $(\mathrm{CL})$ and electron energy loss spectroscopy (EELS) measurements, data processing, STEM, and TEM imaging. R.P.-D. performed the COMSOL simulations on the multipolar decomposition analysis and provided theoretical support of the eigenmode calculation for the true BIC resonance at $\theta=90^{\circ}$. H.W. performed the finite-difference time-domain (FDTD) simulations, including reflectance spectra simulation, $C L$ spectrum simulations, and optical mode patterns calculation. A.F.-D. performed the EELS simulation. S.G. performed the reflectance measurements. S.T.H. provided insightful suggestions to improve the experiments. F.T. and Z.D. performed the chemical vapor deposition (CVD) growth of amorphous Si and inductively coupled plasma (ICP) etching. A.I.K. participated in discussions and gave suggestions. All authors analyzed the data, read and corrected the manuscript before the submission. Z.D. and Z.M. contributed equally to this work.

\section{Conflict of interest}

The authors declare no competing interests.

Supplementary information The online version contains supplementary material available at https://doi.org/10.1038/s41377-021-00707-2.

Received: 26 June 2021 Revised: 1 December 2021 Accepted: 30 December 2021

Published online: 20 January 2022 


\section{References}

1. von Neumann, J. \& Wigner, E. P. Über merkwürdige diskrete Eigenwerte Physikalische Z. 30, 465-467 (1929).

2. Capasso, F. et al. Observation of an electronic bound state above a potential well. Nature 358, 565-567 (1992).

3. Herrick, D. R. Construction of bound states in the continuum for epitaxial heterostructure superlattices. Phys. B+C 85, 44-50 (1976).

4. Stillinger, F. H. Potentials supporting positive-energy eigenstates and their application to semiconductor heterostructures. Phys. B+C 85, 270-276 (1976).

5. Parker, R. Resonance effects in wake shedding from parallel plates: some experimental observations. J. Sound Vib. 4, 62-72 (1966).

6. Linton, C. M. \& Mclver, P. Embedded trapped modes in water waves and acoustics. Wave Motion 45, 16-29 (2007).

7. Lepetit, T. et al. Resonance continuum coupling in high-permittivity dielectric metamaterials. Phys. Rev. B 82, 195307 (2010).

8. Lepetit, T. \& Kanté, B. Controlling multipolar radiation with symmetries for electromagnetic bound states in the continuum. Phys. Rev. B 90, 241103 (2014).

9. Marinica, D. C., Borisov, A. G. \& Shabanov, S. V. Bound states in the continuum in photonics. Phys. Rev. Lett. 100, 183902 (2008).

10. Plotnik, Y. et al. Experimental observation of optical bound states in the continuum. Phys. Rev. Lett. 107, 183901 (2011)

11. Weimann, S. et al. Compact surface fano states embedded in the continuum of waveguide arrays. Phys. Rev. Lett. 111, 240403 (2013).

12. Monticone, F. \& Alù, A. Embedded photonic eigenvalues in 3D nanostructures. Phys. Rev. Lett. 112, 213903 (2014).

13. Hsu, C. W. et al. Observation of trapped light within the radiation continuum. Nature 499, 188-191 (2013).

14. Hsu, C. W. et al. Bound states in the continuum. Nat. Rev. Mater. 1, 16048 (2016).

15. Koshelev, K, Bogdanov, A. \& Kivshar, Y. Engineering with bound states in the continuum. Opt. Photonics N. 31, 38-45 (2020).

16. Liang, $Y$. et al. Bound states in the continuum in anisotropic plasmonic metasurfaces. Nano Lett. 20, 6351-6356 (2020).

17. Jain, A. et al. Electric and magnetic response in dielectric dark states for low loss subwavelength optical meta atoms. Adv. Opt. Mater. 3, 1431-1438 (2015).

18. Fedotov, V. A. et al. Sharp trapped-mode resonances in planar metamaterials with a broken structural symmetry. Phys. Rev. Lett. 99, 147401 (2007).

19. Koshelev, K. et al. Asymmetric metasurfaces with high-Q resonances governed by bound states in the continuum. Phys. Rev. Lett. 121, 193903 (2018).

20. Liu, Z. J. et al. High-Q quasibound states in the continuum for nonlinear metasurfaces. Phys. Rev. Lett. 123, 253901 (2019).

21. Yesilkoy, F. et al. Ultrasensitive hyperspectral imaging and biodetection enabled by dielectric metasurfaces. Nat. Photonics 13, 390-396 (2019).

22. Leitis, A. et al. Angle-multiplexed all-dielectric metasurfaces for broadband molecular fingerprint retrieval. Sci. Adv. 5, eaaw2871 (2019).

23. Rybin, M. V. et al. High-Q supercavity modes in subwavelength dielectric resonators. Phys. Rev. Lett. 119, 243901 (2017).

24. Koshelev, K. et al. Subwavelength dielectric resonators for nonlinear nanophotonics. Science 367, 288-292 (2020).

25. Mylnikov, V. et al. Lasing action in single subwavelength particles supporting supercavity modes. ACS Nano 14, 7338-7346 (2020).

26. Jin, J. C. et al. Topologically enabled ultrahigh-Q guided resonances robust to out-of-plane scattering. Nature 574, 501-504 (2019).

27. Yin, X. F. et al. Observation of topologically enabled unidirectional guided resonances. Nature 580, 467-471 (2020).

28. Ha, S. T. et al. Directional lasing in resonant semiconductor nanoantenna arrays. Nat. Nanotechnol. 13, 1042-1047 (2018).

29. Huang, C. et al. Ultrafast control of vortex microlasers. Science 367, 1018-1021 (2020).

30. Wu, M. F. et al. Room-temperature lasing in colloidal nanoplatelets via mieresonant bound states in the continuum. Nano Lett. 20,6005-6011 (2020).

31. Carletti, L. et al. Giant nonlinear response at the nanoscale driven by bound states in the continuum. Phys. Rev. Lett. 121, 033903 (2018).

32. Bernhardt, N. et al. Quasi-BIC resonant enhancement of second-harmonic generation in WS 2 monolayers. Nano Lett. 20, 5309-5314 (2020).
33. Gorkunov, M. V., Antonov, A. A. \& Kivshar, Y. S. Metasurfaces with maximum chirality empowered by bound states in the continuum. Phys. Rev. Lett. 125 , 093903 (2020).

34. Tittl, A. et al. Imaging-based molecular barcoding with pixelated dielectric metasurfaces. Science 360, 1105-1109 (2018).

35. Kodigala, A. et al. Lasing action from photonic bound states in continuum. Nature 541, 196-199 (2017).

36. Bosman, M. et al. Mapping surface plasmons at the nanometre scale with an electron beam. Nanotechnology 18, 165505 (2007).

37. Dong, Z. G. et al. Fabrication of suspended metal-dielectric-metal plasmonic nanostructures. Nanotechnology 25, 135303 (2014).

38. Duan, H. G. et al. Nanoplasmonics: classical down to the nanometer scale Nano Lett. 12, 1683-1689 (2012).

39. Kociak, M. \& Stéphan, O. Mapping plasmons at the nanometer scale in an electron microscope. Chem. Soc. Rev. 43, 3865-3883 (2014).

40. García de Abajo, F. J. Optical excitations in electron microscopy. Rev. Mod. Phys. 82, 209-275 (2010).

41. Zheng, S. J. et al. Giant enhancement of cathodoluminescence of monolayer transitional metal dichalcogenides semiconductors. Nano Lett. 17, 6475-6480 (2017).

42. Losquin, A. \& Kociak, M. Link between cathodoluminescence and electron energy loss spectroscopy and the radiative and full electromagnetic local density of states. ACS Photonics 2, 1619-1627 (2015).

43. Coenen, T. et al. Cathodoluminescence microscopy: optical imaging and spectroscopy with deep-subwavelength resolution. MRS Bull. 40, 359-365 (2015).

44. Knight, M. W. et al. Aluminum plasmonic nanoantennas. Nano Lett. 12, 6000-6004 (2012)

45. Bischak, C. G. et al. Cathodoluminescence-activated nanoimaging: noninvasive near-field optical microscopy in an electron microscope. Nano Lett. 15 3383-3390 (2015).

46. Han, T. Y. et al. Reveal and control of chiral cathodoluminescence at subnanoscale. Nano Lett. 18, 567-572 (2018).

47. Sannomiya, T. et al. Cathodoluminescence phase extraction of the coupling between nanoparticles and surface plasmon polaritons. Nano Lett. 20, 592-598 (2020).

48. Coenen, T. \& Polman, A. Optical properties of single plasmonic holes probed with local electron beam excitation. ACS Nano 8, 7350-7358 (2014).

49. Zu, S. et al. Imaging of plasmonic chiral radiative local density of states with cathodoluminescence nanoscopy. Nano Lett. 19, 775-780 (2019).

50. Coenen, T., van de Groep, J. \& Polman, A. Resonant modes of single silicon nanocavities excited by electron irradiation. ACS Nano 7, 1689-1698 (2013).

51. van de Groep, J. et al. Direct imaging of hybridized eigenmodes in coupled silicon nanoparticles. Optica 3, 93-99 (2016).

52. Marino, G. et al. Cathodoluminescence imaging spectroscopy of single and dimer AlGaAs nano-disks. In European Conference on Lasers and Electro-Optics and European Quantum Electronics Conference, CK_13_2 (OPTICA Publishing Gr) (2017) https://www.osapublishing.org/abstract. cfm?uri=CLEO Europe-2017-CK_13_2.

53. Peng, S. Y. et al. Probing the band structure of topological silicon photonic lattices in the visible spectrum. Phys. Rev. Lett. 122, 117401 (2019)

54. Sapienza, R. et al. Deep-subwavelength imaging of the modal dispersion of light. Nat. Mater. 11, 781-787 (2012).

55. Murai, S. et al. Bound states in the continuum in the visible emerging from out-of-plane magnetic dipoles. ACS Photonics 7, 2204-2210 (2020).

56. Das, P., Chini, T. K. \& Pond, J. Probing higher order surface plasmon modes on individual truncated tetrahedral gold nanoparticle using cathodoluminescence imaging and spectroscopy combined with FDTD simulations. J. Phys. Chem. C 116, 15610-15619 (2012)

57. Dong, Z. G. et al. Ultraviolet interband plasmonics with Si nanostructures Nano Lett. 19, 8040-8048 (2019).

58. Bosman, M. \& Keast, V. J. Optimizing EELS acquisition. Ultramicroscopy $\mathbf{1 0 8}$ 837-846 (2008)

59. Dong, Z. G. et al. Printing beyond SRGB color gamut by mimicking silicon nanostructures in free-space. Nano Lett. 17, 7620-7628 (2017). 